\title{
CUÁNDO OPERAR UN PACIENTE CON SEPSIS EN ESTADO CRÍTICO
}

\author{
Mario Gómez Duque MD FCCM*, Hedda María Uribe Guzmán MD**, Juan David Lleras Riaño MD***
}

\section{Resumen}

La infección intraabdominal de cualquier origen es la segunda causa de muerte por sepsis en los pacientes en estado crítico y continúa siendo un reto para el médico tratante, dado que existen muchos factores que afectan el enfoque del manejo racional. La creciente tasa de resistencia de los gérmenes intrahospitalarios a las terapias antibióticas convencionales, las diferentes visiones que existen sobre cómo dilucidar el dilema de definir una conducta médica o quirúrgica y la falta de ensayos clínicos con suficiente validez que nos proporcionen una guía que cambie el curso de la enfermedad, hacen de este problema un objeto de constante discusión y discrepancia entre los especialistas que intervienen en su solución. Esta revisión actualiza los conceptos de grupos multidisciplinarios que ayudarán a definir la conducta para el manejo apropiado de esta grave situación clínica.

Palabras clave: sepsis, paciente crítico, infección intraabdominal, pronóstico, mortalidad.

Abreviaturas: UCI, unidad de cuidado intensivo.

\section{WHEN TO OPERATE A SEPTIC CRITICALly ILL PATIENT}

\begin{abstract}
Regardless its origin, intra-abdominal infection is the second cause of death due to sepsis in critically ill patients and remains a challenge for the treating physician due to the many factors affecting the approach of a rational management. The increase of the resistance rate to conventional antimicrobial therapy of nosocomial organisms, different opinions on how to work out the dilemma of defining a medical or surgical treatment and the lack of valid clinical trials providing guidelines which change the disease's course, makes this problem a subject of constant discussion and discrepancy between intervening specialists. This review updates the concepts of various multidisciplinary groups which will help define the conduct for appropriate handling of this serious clinical condition.
\end{abstract}

Key words: sepsis, critically ill patient, intra-abdominal infection, prognosis, mortality rate.

Fecha recibido: diciembre 10 de 2010 - Fecha aceptado: mayo 27 de 2011

* Profesor Titular de Medicina Crítica, Fundación Universitaria de Ciencias de la Salud. Jefe Unidades de Cuidado Intensivo Hospital de San José, Infantil Universitario de San José y Clinica Fundadores. Bogotá DC, Colombia.
** Residente III, Cirugía General, Fundación Universitaria de Ciencias de la Salud. Hospital de San José. Bogotá DC, Colombia.

*** Residente II año Cirugía General, Fundación Universitaria de Ciencias de la Salud. Hospital de San José. Bogotá DC, Colombia. 


\section{Introducción}

No podrá aplicarse con más contundencia el famoso aforismo del cirujano que describe en forma magistral el dilema: la segunda decisión más difícil para un cirujano es cuándo operar un paciente, porque la decisión más difícil es cuándo reoperarlo.

Desde comienzos del siglo pasado se ha centrado el manejode la sepsis abdominal en la erradicación del foco, principio que sigue siendo vigente y que no ha sido el único que ha disminuido la mortalidad en estos casos, sino que desde el punto de vista técnico quirúrgico ha motivado la aparición de diferentes alternativas para un mejor manejo de estos pacientes. Durante las últimas tres décadas, el enfoque multidisciplinario e integral ha contribuido de manera importante a mejorar los resultados. Las técnicas de control de daños y la prevención de la tríada de la muerte (hipotermia, acidosis e hipoperfusión) como la laparostomía abdominal y el advenimiento de alternativas menos invasivas (drenajes percutáneos dirigidos por imágenes, sistemas de "vacum" permanente) han complementado el tratamiento. ${ }^{1}$ Los avances en el conocimiento de la fisiopatología de la enfermedad, la aparición de nuevos antibióticos y la introducción de novedosas tecnologías diagnósticas y de monitoría del paciente crítico, han generado cambios sustanciales en el abordaje, logrando una disminución significativa de la mortalidad de $90 \%$ cuando el manejo era solo médico, hasta $20 \%$ a $30 \%$ en la actualidad. Este trabajo pretende describir los conceptos actuales desde la visual de un grupo multidisciplinario de trabajo, que permita a los profesionales tener alguna claridad para el manejo apropiado de esta compleja patología. ${ }^{2}$

\section{Aspectos históricos}

A principios del siglo XX Alfred Blalock describió el choque como una entidad que reflejaba un déficit de volumen intravascular absoluto o relativo, dando inicio a los conceptos de soporte y resucitación hemodinámica. ${ }^{3}$ El doctor Samuel Gross veinte años antes definió el estado de choque como un severo desacople de la maquinaria de la vida. Desde esa época se ha identificado una estrecha relación entre la función hemodinámica y la oxigenación tisular. Los cambios hemodinámicos relacionados con los estados de sepsis son la consecuencia final del mal pronóstico en estos casos.

El manejo quirúrgico de la infección se conoce desde la antigüedad. El drenaje de abscesos se describe en papiros y documentos egipcios, babilonios y romanos. Para el siglo XV, Amboise Paré propuso el manejo de heridas quirúrgicas infectadas y realizó drenaje de abscesos intracavitarios, pero solo a mediados del siglo XIX se aceptó esta conducta al identificar los gérmenes que causaban dichas patologías. ${ }^{3}$ Kishner fue quien introdujo los tres principios básicos del tratamiento quirúrgico de esta afección: eliminación del foco, extirpación del tejido necrótico y drenaje del material purulento, contribuyendo así a la comprensión de la fisiopatología y por supuesto también a un descenso importante en la morbimortalidad. ${ }^{2}$

Otro factor que ayudó a disminuir la mortalidad fue la introducción de los antibióticos. Fleming descubrió la actividad antibacteriana de la penicilina hacia 1920 y a principios del siglo XX se utilizó como parte de la terapia en la peritonitis. ${ }^{3}$ Después hacia la década del setenta aparecen nuevas moléculas más complejas, de mayor espectro, como las cefalosporinas y luegolas quinolonas, los carbapenémicos y los blactámicos. ${ }^{2}$

Las pautas de ratamiento actual en sepsis grave y choque séptico resaltan conceptos reconocidos desde hace varias décadas como son el drenaje del foco séptico, inicio temprano de antibióticos, (en las primeras seis horas después de la identificación del foco séptico), toma de cultivos y reanimación por metas. ${ }^{4}$ Los criterios de seguimiento en la UCI con una patología abdominal de origen séptico, como la de todos los pacientes críticos, incluyen una valoración secuencial por sistemas que orientan el manejo momento a momento.

Las causas de inestabilidad son múltiples y la evidencia de una quirúrgica no siempre es clara ni evidente. El estado crítico denota muchas circunstancias que hacen perder la objetividad de la evaluación clínica y los estados de hipoperfusión, aún los causados por un foco séptico, son multifactoriales. La sensibilidad diagnóstica de las imágenes no permite, ni siquiera con la ayuda de la tomografía, apoyar un diagnóstico seguro. Todas estas 
circunstancias son causa de la discrepancia y muchas veces la sospecha subjetiva de un foco séptico por la presencia de una hipoperfusión que persiste a pesar de una reanimación adecuada, constituyen el único argumento que soporta la indicación de una exploración quirúrgica. $^{5}$

En un intento por dilucidar el problema, nuestro grupo de trabajo desarrolló un score o valoración de reintervención quirúrgica (IRQUI) originado en un análisis retrospectivo de 180 pacientes manejados durante dos años en las UCI del Hospital de San José y la Clínica Fundadores, buscando mediante un análisis de regresión logística los factores que contribuyeron al peor desenlace y a los que se les asignó un mayor puntaje. Estos fueron hiperdinamia persistente, taquicardia, demora de más de 48 horas en la decisión quirúrgica ante la sospecha de un foco séptico persistente, presencia en los cultivos de líquido peritoneal de gérmenes saprofitos, multirresistencia antibiótica y la disfunción multiorgánica. ${ }^{6}$ Este desacuerdo podría dirimirse a la luz de la medicina basada en la evidencia, pero no hay en la literatura actual ensayos que con claridad ayuden a resolver el problema de cuándo operar o reoperar un paciente. ${ }^{2}$

\section{Metodología}

Se realizó una revisión sistemática de la literatura para identificar las pautas actuales que definan el momento apropiado para la reoperación abdominal en pacientes sépticos en estado crítico. Para tal efecto se analizaron las publicaciones más relevantes de las últimas dos décadas.

\section{Marco teórico}

\section{Peritoneo: anatomía y fisiología}

El peritoneo fue visto en la antigüedad como una estructura aislada sin una función determinada; sin embargo, en la actualidad se conoce como un órgano con múltiples funciones como la de lubricar los órganos intraabdominales, censar la presencia de virus y bacterias, la absorción de partículas y el control y la regulación inmunológica. ${ }^{7}$
La cavidad abdominal se encuentra cubierta por una membrana de tejido conjuntivo llamada peritoneo, que por su disposición puede ser visceral cuando envuelve los órganos contenidos en el abdomen o parietal cuando se encuentra tapizando la pared abdominal. Está compuesto por una matriz de fibras de colágeno donde se disponen células mesoteliales, adiposas, linfocitos, macrófagos y fibroblastos. Su función primordial es el intercambio de agua, solutos y partículas de mayor tamaño, con una superficie de intercambio de cerca de un metro cuadrado, para una total de $1,72 \mathrm{~m}^{2}$. La eliminación de la mayor parte de sustancias y partículas así como de microorganismos se realiza a nivel de las lagunas diafragmáticas, que son una serie de estomas localizadas en la región subdiafragmática que están asociadas con una red linfática y drenan en el conducto torácico a través de un mecanismo de bomba generado por la contracción y relajación del diafragma durante el proceso de la ventilación. De esta manera una partícula que se encuentre en la cavidad peritoneal pasará al conducto torácico en cerca de seis minutos y a la circulación sanguínea en doce minutos. ${ }^{2}$

\section{Peritonitis}

La peritonitis es un proceso inflamatorio difuso que compromete toda la cavidad. Hace referencia a un conjunto de circunstancias que ocurren luego de la contaminación del espacio intraperitoneal como resultado de otra patología. Puede clasificarse según su patogenia en primaria, secundaria o terciaria. ${ }^{2,8}$ La primaria aparece como resultado de la diseminación hematógena de una bacteria al espacio peritoneal desde un órgano remoto. La secundaria es la consecuencia de la contaminación del peritoneo por la colonización o la infección de un órgano intraabdominal, ya sea por la perforación de una víscera hueca, el drenaje de un absceso de un órgano sólido o el escape de fluido contaminado a ravés de una anastomosis o fístula. La causa más común de la secundaria bacteriana es la apendicitis perforada, pero no es una causa frecuente de peritonitis terciaria. ${ }^{8}$

La peritonitis terciaria es una inflamación infecciosa recurrente o persistente luego de una secundaria. ${ }^{8} \mathrm{Su}$ severidad es mayor que la de las anteriores y por lo regular se diferencia por su persistencia y los tipos de 
gérmenes que la perpetúan. La mayoría de las veces se origina en un foco no controlado u oculto. ${ }^{9}$ Según el último consenso de definiciones de infección en cuidado intensivo, la peritonitis terciaria es una infección intraabdominal que persiste o recurre después de más 48 horas del control quirúrgico de la fuente que en apariencia fue adecuada y exitosa. ${ }^{10}$ Se manifiesta como una respuesta inflamatoria prolongada o falla orgánica múltiple, con una mortalidad entre 30 y $65 \%$. Dentro de la microflora asociada con esta patología se encuentran bacterias nosocomiales, oportunistas facultativas y hongos en su mayoría multirresistentes que requieren terapia antibiótica de amplio espectro. ${ }^{10}$

La gravedad de la enfermedad depende de varios factores como el origen, volumen y duración del inóculo, y la respuesta local y sistémica del paciente. "Son factores clave para la severidad de la infección en peritonitis secundaria ${ }^{8}$ :

- El grado de contaminación bacteriana.

- La virulencia de la bacteria involucrada y los efectos sinérgicos asociados.

- La presencia de factores adyuvantes como contenido intestinal, sangre, bilis, bario, etc.

- La integridad de la respuesta del huésped tanto local como sistémica.

- El tratamiento adecuado.

La peritonitis primaria es una infección casi siempre monobacteriana. La secundaria se asocia con flora mixta y la presencia de anaerobios obligados. El concepto de infección bifásica hace referencia a la presencia de bacterias que se potencian en forma sinérgica, como es el caso de la Escherichia coli con un anaerobio facultativo, que sería responsable de la fase aguda de la peritonitis y el Bacteroides fragilis, con un anaerobio obligado, responsable de la formación tardía de abscesos. ${ }^{12}$ Sin embargo, la mayoría de los pacientes presentan tanto microorganismos aerobios como anaerobios. Los multirresistentes suelen encontrarse en peritonitis terciaria y son un factor de falla del tratamiento. $^{8}$
El contactodel peritoneo con un microorganismo genera un aumento en la filtración de líquido peritoneal, con producción de factores quimiotácticos que producen al principio una respuesta inflamatoria local. Este estímulo genera tres respuestas frente a la infección: ${ }^{2}$

- Eliminación mecánica del agente a través del drenaje linfático por las lagunas diafragmáticas, lo que causa un importante inóculo bacteriano a distancia que puede llevar a bacteremia, sepsis y choque.

- Liberación de líquido peritoneal rico en proteínas y macrófagos (exudado), causando un aumento en la presión oncótica en la cavidad que puede facilitar la formación de un tercer espacio.

- Liberación de catecolaminas, hormonas de la corteza suprarrenal, aldosterona y muchas otras que desencadenan la respuesta sistémica que acompaña a la enfermedad.

Una vez implantado un germen en la cavidad peritoneal se inicia la actividad celular local que junto con mecanismos de contención, como el desplazamiento del epiplón al área afectada, buscan controlar la infección. Cuando esto no se logra, ya sea por la pobre respuesta de las defensas del huésped o porque el inóculo sobrepasa la capacidad de las mismas, se desencadena una respuesta inflamatoria generalizada dando como resultado el patrón conocidocomo peritonitis, que en cuestión de minutos llevará a su vez a una respuesta inflamatoria sistémica por el paso de las bacterias a la sangre y a la vía linfática, lo más probable a través de las manchas lechosas de Ranvier. $^{13,14}$

La diseminación sistémica que se produce por los cambios en la permeabilidad de la membrana de la lámina propia y permite el paso tanto de bacterias como de endotoxinas, se ha denominado inflamación intravascular maligna, que es la entidad que perpetúa la lesión orgánica a distancia y empeora el pronóstico. ${ }^{15-17}$ Las células mesoteliales y sus interacciones con los macrófagos peritoneales juegan un papel esencial en la respuesta inicial a la infección durante el reclutamiento leucocitario y en la fase de resolución de la inflamación.

$\mathrm{Al}$ ingresar una bacteria a la cavidad y hacer contacto con el peritoneo visceral o parietal, las células mesoteliales 
del mismo, pueden lesionarse o fagocitar la bacteria e iniciar la secreción de IL-8. Por otro lado, la presencia de bacterias y sus productos estimulan la producción de IL-2 y TNFalfa por parte de los macrófagos peritoneales, generando la producción de citoquinas y eicosanoides. ${ }^{7}$ Los macrófagos locales liberan un serie de citoquinas proinflamatorias dentro de las que se encuentran TNF, IL-1, IL-6, y IL-8, las cuales estimulan las propiedades bactericidas de los leucocitos y la fagocitosis. Además de generar un aumento en el reclutamiento de células fagocíticas, producen una elevación de la agregación leucocitaria en el endotelio con liberación de proteasas y metabolitos del ácido araquidónico, en especial por efecto del TNF-alfa y la interleucina $1 . .^{8,11,18,19} \mathrm{El}$ control de la liberación de IL-6 ha demostrado disminución en la severidad de la lesión en algunos ensayos clínicos. ${ }^{8}$

La respuesta inflamatoria mediada por el complemento genera acúmulos de fibrina en el espacio peritoneal por el exudado fibrinoso que aparece, con el fin de generar el secuestro bacteriano que bloquea en forma parcial la absorción subdiafragmática del líquido peritoneal, llevando a acúmulo de líquido, bacterias, membranas fibrinosas y productos de degradación en la cavidad abdominal. La IL-8 es un potenteestimulante de los neutrófilos, se libera en las primeras 24 horas del proceso inflamatorio y es por eso que el tipo celular predominante en ese momento son los neutrófilos. Después, se inicia la liberación de sustancias que atraen a los monocitos, convirtiéndolas en las más abundantes luego de las 24 horas. ${ }^{7}$ Existen algunos agentes que facilitan la infección intraabdominal como la presencia de sangre, bilis, secreciones gástrica y pancreática, y orina, quilo o cuerpos extraños en la cavidad. . $^{130-22}$

Las colecciones intraabdominales por lo regular se encuentran cubiertas por una cápsula de colágeno que contiene además un anillo de leucocitos que mantiene aisladas a las bacterias, impidiendo la invasión al resto del organismo, lo cual se conoce como absceso. Sin embargo, el centro del absceso es un área inhabitable para los leucocitos ya que presenta un $\mathrm{pH}$ ácido, baja concentración de fluido con opsoninas, pobres niveles de oxígeno, tejido necrótico, enzimas protreolíticas y gran cantidad debacterias que impiden el control de la infección por el sistemainmune. ${ }^{13}$
La respuesta inflamatoria peritoneal es perpetuada por la presencia de fibrina, tejido necrótico y bacterias, asociados con un constante influjo de leucocitos y la liberación de sustancias proinflamatorias. Después la infección puede curarse pero el proceso inflamatorio peritoneal persiste, lo cual es difícil de identificar en la clínica, haciendo imposible distinguir los pacientes que se beneficiarían con una cirugía por la persistencia del foco séptico, de los que solocontinúan con el proceso inflamatorio peritoneal pero sin infección. ${ }^{7}$ El manejo de esta patología debe ser entusiasta, rápido y completo, pues la idea es evitar la formación de óxido nítrico por medio del sistema de la óxido nítrico sintetasa inducible, que produce disminución de la motilidad intestinal, además de lesión celular y endotelial debido al bloqueo del ciclo de Krebs. ${ }^{12}$

\section{Definición de términos: infección}

El concepto de infección instaurada fue descrito por Miles y Burke como el período decisivo (Londres, 1967) en el que el crecimiento bacteriano sobrepasa un volumen de 103 bacterias por $\mathrm{cm}^{3}$ de tejido o fluido corporal. ${ }^{13} \mathrm{La}$ respuesta del sistemainmune en el individuo sano mantiene el crecimiento bacteriano por debajo de este umbral evitando así la infección. La caída del inóculo bacteriano se facilita por la presencia de complemento y anticuerpos, que causan un aumento de la permeabilidad endotelial a los polimorfonucleares, los cuales requieren del oxígeno para generar la respuesta contra las bacterias y en consecuencia se aumenta el flujo tisular de estos. Es por estoque la adecuada reanimación volumétrica restaurando la perfusión tisular ayuda al control de la fuente, así como el adecuado drenaje de las colecciones y el desbridamiento del tejido no viable. ${ }^{13}$

\section{Infección intraabdominal}

Este concepto engloba un conjunto de procesos infecciosos heterogéneos que comparten la localización anatómica entre el diafragma y la pelvis. ${ }^{10}$ Es la inflamación de cualquier órgano intraabdominal y se considera como no complicada mientras permanezca intramural. Este tipo de infecciones se encuentran en capacidad de progresar a una complicada cuando no son tratadas en forma oportuna y adecuada con la posterior repercusión sistémica. ${ }^{1}$ Peritonitis e infección 
intraabdominal no son sinónimos, pues la primera hace referencia a la inflamación del peritoneo por cualquier causa, mientras la segunda es la peritonitis secundaria a una inoculación bacteriana que trae como resultado la respuesta inflamatoria sistémica. ${ }^{12}$ Algunos autores consideran que la peritonitis hace referencia desde un punto de vista local al síndrome de respuesta inflamatoria sistémica y la infección intraabdominal es la forma local de una sepsis. ${ }^{1,11}$

Las infecciones intraabdominales pueden clasificarse en localizadas o diseminadas. Las primeras son colecciones o abscesos cuya ubicación varía según el origen, pudiéndose encontrar en los espacios subfrénicos, subhepático, el fondo de saco, entre asas intestinales, el receso pélvico o en el parénquima de cualquier órgano sólido. Por otro lado, las diseminadas en general se presentan como peritonitis generalizada comprometiendo la mayoría de la cavidad peritoneal y son el resultado de falla en la respuesta inmune local o su ausencia. ${ }^{13} \mathrm{El}$ término infección intraabdominal complicada hace referencia a la extensión al espacio peritoneal desde su sitio de origen, incluyendo peritonitis o la formación de abscesos, sin tener en cuenta la severidad del cuadro., ${ }^{10}$

Múltiples factores se encuentran relacionados con la severidad de la infección, dentro de los cuales se encuentran la fuente anatómica y el grado de compromiso fisiológico secundario. El APACHE ha sido validado por la Sociedad de Infección Quirúrgica (Surgical Infection Society) como el mejor método de estratificación del riesgo de infección. ${ }^{12}$

La falla en los mecanismos de control de la infección está asociada con: ${ }^{7}$

1. Grandes acúmulos de exudado libre rico en proteínas en la cavidad peritoneal que impide la opsonización bacteriana por la disolución, disminuyendo la capacidad de los fagocitos para destruir las bacterias.

2. La presencia de sustancias adyuvantes de la infección (bilis, sangre, cuerpos extraños) que diluyen las citoquinas y las opsoninas. Además, la fibrina inhibe la migración fagocitaria e impide la fagocitosis de la E. coli por los neutrófilos.
3. Las sustancias adyuvantes se asocian con el aumento en la proliferación y virulencia bacterianas.

4. Sinergismo entre especies bacterianas.

5. La fibrina provee un ambiente seguro para las bacterias, donde pueden proliferar sin ser afectadas por los neutrófilos.

\section{Sepsis, sepsis grave y choque séptico}

La sepsis se define como las manifestaciones sistémicas asociadas con una infección (Tabla 1). Se considera grave si la anterior se acompaña de disfunción de un órgano o hipoperfusión tisular secundaria (Tabla 2). Se define choque séptico cuando a lo anterior se agrega una tensión arterial media menor a $70 \mathrm{~mm} / \mathrm{Hg}$ o una presión arterial sistólica menor de $90 \mathrm{~mm} / \mathrm{Hg}$ o una caída en la misma mayor de $40 \mathrm{~mm} / \mathrm{Hg}$. ${ }^{4}$

\section{Factores de riesgo}

La severidad de la infección intraabdominal se ha visto relacionada con ciertas circunstancias que serán enumeradas en la Tabla 3.

\section{Diagnóstíco}

Desde el punto de vista clínico se caracteriza por un cuadro de dolor abdominal de inicio agudo o insidioso en el $100 \%$ de los pacientes, por lo regular asociado con náusea y anorexia. $\mathrm{Al}$ examen físico se encuentra un paciente de apariencia tóxica, con fiebre superior a $40^{\circ} \mathrm{C}$ o hipotermia, en posición antálgica que puede corresponder a la de tipo fetal o en decúbito supino con los miembros inferiores en flexión hacia el abdomen. En el paciente séptico se puede evidenciar estado hiperdinámico con un alto gasto cardíaco y resistencia vascular periférica disminuida, evidenciada a la palpación por el pulso saltón con amplitud variable según el estado del volumen circulante. Se puede identificar el compromiso temprano de la válvula pulmonar por la presencia de un soplo sistólico acompañado de thrill, de mayor intensidad auscultable en el área pulmonar y que es secundario a la presencia de una estenosis relativa de la válvula por hipertensión pulmonar aguda (complejo de 


\section{Tabla I. Criterios diagnósticos para sepsis}

Infección documentada o sospechada, más alguno de los siguientes:

\section{Variables generales}

- Fiebre $\left(38^{\circ} \mathrm{C}\right)$ o hipotermia $\left(36^{\circ} \mathrm{C}\right)$.

- Taquicardia > de 90/min.

- Taquipnea.

- Alteración del estado mental.

- Balance de fluidos muy positivo (20 cc/k/24 h en 24 horas).

- Hiperglicemia en ausencia de diabetes (glucosa plasmática) $140 \mathrm{mg} / \mathrm{dL}$

\section{Variables inflamatorias}

- Leucocitosis (conteo de leucocitos mayor de $12.000 \mathrm{mcL}-1$ ).

- Leucopenia (conteo de leucocitos menor de $4.000 \mathrm{mcL}-1$ ).

- Presencia de formas inmaduras mayor de $10 \%$ en un hemograma normal.

- Proteína $C$ reactiva plasmática $2 \mathrm{DE}$ por encima del valor normal.

- Procalcitonina plasmática 2 DE por encima del valor normal

\section{Variables hemodinámicas}

- Hipotensión arterial (TAM 70 mm/Hg, TAS 90 mm/Hg o disminución superior a $40 \mathrm{~mm} / \mathrm{Hg}$ ).

\section{Variables de disfunción orgánica}

- Hipoxemia (Pa02/FIO2 300).

- Oliguria (gasto urinario $0.5 \mathrm{cc} / \mathrm{k} / \mathrm{h}$ por lo menos en 2 horas a pesar de una adecuada reanimación volumétrica).

- Aumento en valores de creatinina > a $0.5 \mathrm{mg} / \mathrm{dL}$.

- Trastorno de la coagulación (INR > 1.5 o PTT > a $60 \mathrm{seg}$ ).

- Íleo (ausencia de ruidos intestinales).

- Trombocitopenia (conteo de plaquetas 100,000 mcL-I).

- Hiperbilirrubinemia (bilirrubina plasmática total $4 \mathrm{mg} / \mathrm{dL}$ )

\section{Variables de perfusión tisular}

- Hiperlactatemia.

- Llenado capilar retardado o piel moteada.

Adaptado de: Dellinger R, Levy M, Carlet J. Surviving Sepsis Campaign: International guidelines for management of severe sepsis and septic shock: 2008, Crit Care Med 2008 (I-33).

\section{Tabla 2. Criterios diagnósticos para sepsis severa}

Sepsis severa es la asociada con hipoperfusión tisular inducida o disfunción de un órgano dado por uno de los siguientes:

- Hipotensión inducida por sepsis.

- Lactato aumentado.

- Oliguria a pesar de una reanimación volumétrica adecuada.

- Lesión pulmonar aguda con PaO2/FIO2 menor de 200 en presencia de neumonía como fuente de infección.

- Creatinina > de $2 \mathrm{mg} / \mathrm{dL}$.

- Bilirrubina $>$ de $2 \mathrm{mg} / \mathrm{dL}$.

- Trombocitopenia.

- Coagulopatía.

Adaptado de: Dellinger R, Levy M, Carlet J. Surviving Sepsis Campaign: International guidelines for management of severe sepsis and septic shock: 2008, Crit Care Med 2008 (I-33).

Tabla 3. Factores predictores de falla en el control de la fuente de infección intraabdominal

- Retraso en la intervención inicial (> 24 horas).

- Enfermedad severa (APACHE II > 15).

- Edad avanzada.

- Comorbilidades y grado de disfunción orgánica asociada.

- Bajos niveles de albúmina.

- Pobre estado nutricional.

- Grado de compromiso peritoneal o peritonitis generalizada.

- Incapacidad de realizar un adecuado drenaje y desbridamiento del foco infeccioso.

- Presencia de malignidad.

Adaptado de: Solomkin JS, Mazuski JE. Intra-abdominal Sepsis: Newer Interventional and Antimicrobial Therapies. Infect Dis Clin N Am 23 (2009) 593-608. 
la pulmonar de Chavez). ${ }^{15,23-26} \mathrm{El}$ examen abdominal evidencia dolor abdominal difuso, pero con un punto de máximo dolor que por lo regular corresponde a la localización anatómica de la lesión inicial.?

Los pacientes críticos que presentan una infección severa intraabdominal son difíciles de valorar ya que por lo general están en estado de choque, se encuentran con alteración del estado de conciencia o con ventilación mecánica asociada. Casi siempre el examen físico es inespecífico y antes de decidir una laparotomía hay obligación de descartar otras fuentes de infección como sinusitis, absceso perianal, úlceras infectadas, flebitis o infección de vías aéreas. Si la evaluación inicial sugiere un foco abdominal debe realizarse un abordaje sistemático priorizando los recursos a favor del paciente y en contra del tiempo, de forma tal que se enfatizará en las ayudas diagnósticas más relevantes y que no pongan en riesgo la vida del paciente, como transportarlo a una TAC con altos parámetros de ventilación mecánica o inestabilidad hemodinámica. En estos casos se llevará a laparotomía diagnóstica, con el fin de confirmar la sospecha de infección intraabdominal y la búsqueda subsecuente de la causa. ${ }^{7.27,28}$

\section{Manejo médico}

Siempre deben descartarse causas no quirúrgicas de dolor abdominal. Se inicia el protocolo de reanimación en el momento mismo del diagnóstico, en especial si se encuentra en choque séptico, teniendo como metas las siguientes:

- Presión venosa central entre 8 y 12 mm/Hh Hg (recomendación 1C).

- Presión arterial media mayor de $65 \mathrm{~mm} / \mathrm{Hg}$ (recomendación 1C).

- Gasto urinario mayor o igual a $0,5 \mathrm{cc} / \mathrm{k} / \mathrm{h}$.

- Saturación venosa central mayor de $70 \%$.

El primer paso es la resucitación volumétrica con cristaloides ya que todos presentan algún grado de hipovolemia, iniciando con la infusión de dos litros de solución cristaloide a través de dos accesos venosos periféricos de calibre amplio, en quienes que no presenten signos de falla renal o cardíaca. Durante este paso deberá instaurarse el monitoreo de signos vitales, incluyendo el examen del estado mental y el gasto urinario con el fin de vigilar la perfusión distal. Para los casos severos de pacientes en la UCI se recomienda la resucitación volumétrica cuidadosa dirigida por monitoreo hemodinámico con medición del gasto cardíaco continuo. Cerca del $50 \%$ no responde a una carga de volumen aumentando cl gasto cardíaco y por lo tanto habrá que modificar la conducta terapéutica para evitar la sobrecarga y el empeoramiento del compromiso pulmonar. ${ }^{29-32}$ En los casos críticos con múltiples comorbilidades y en ancianos se debe considerar monitorización invasiva, como es la cateterización venosa central y la línea arterial. ${ }^{7,33}$

Las principales recomendaciones del Surviving Sepsis Campaign 2008 son: $^{4}$

- Uso de cristaloides o coloides en la resucitación (recomendación 1B).

- La infusión de cristaloides debe ser de $1.000 \mathrm{ml}$ en 30 minutos o 300 a $500 \mathrm{ml}$ de coloides en el mismo tiempo (recomendación 1D).

- La dopamina y la noradrenalina son los vasopresores de elección y se deberán administrar por un acceso venosa central (recomendación 1C).

- No usar dosis bajas de dopamina para protección renal (recomendación lA).

- Instaurar monitoreo invasivo cuando se requiere manejo vasopresor (recomendación 1D).

- Aplicardobutamina ante disfunción miocárdica dada por elevadas presiones de llenado y bajo gasto cardíaco (recomendación 1C).

- Considerar el empleo de hidrocortisona cuando no respondan a resucitación volumétrica y vasopresores (recomendación 2C) a dosis menores a $300 \mathrm{mg}$ por día (recomendación 1A).

- No recurrir a corticoides en sepsis sin estado de choque (recomendación 1D).

\section{Manejo nutricional}

Cuando hay infección intraabdominal, en especial en los pacientes en choque secundario, se presenta una 
respuesta metabólica que puede dividirse en dos fases: la catabólica (fase "ebb" del choque) caracterizada por un gasto energético normal o disminuido, hipoglicemia y disminución de la perfusión tisular, y la anabólica (fase "flow" del choque) caracterizada por hipermetabolismo, balance negativo de nitrogenados e hiperglicemia. Hoy día se reconoce que las dos fases se sobreponen. La fiebre secundaria a la presencia de IL-I genera un aumento en la tasa metabólica. La respuesta hormonal al estrés está dada por liberación de cortisol, ACTH, catecolaminas, hormona de crecimiento, glucagón y arginina-vasopresina. Estos cambios sumados a los niveles elevados de citoquinas proinflamatorias generan una alteración del metabolismo de carbohidratos, lípidos y proteínas.

Los bajos niveles de glutamina se asocian con un peor pronóstico, debido al aumento de la traslocación bacteriana y el síndrome de disfunción multiorgánica, pues este aminoácido es una fuente esencial de energía para el intestino, los linfocitos y los fibroblastos y además juega un papel importante en la restauración del sistema inmune y la barrera mucosa a nivel gastrointestinal. Por esta razón es importante la administración por vía enteral de glutamina, que es superior a la endovenosa. ${ }^{7,34,35}$ A pesar de la controversia, recomendamos la nutrición enteral temprana pues nuestra experiencia ha demostrado una disminución en la mortalidad, las complicaciones y el costo. ${ }^{36.37}$

\section{Antibioticoterapia}

Debe iniciarse durante la primera hora del diagnóstico de sepsis severa (recomendación 1D) y choque séptico (recomendación 1B). Se comienza como manejo empírico con uno o dos antibióticos que cubran la flora sospechosa y tengan buena penetrancia a la fuente de infección (recomendación 1B). La duración del esquema terapéutico debe ser de siete a diez días y solo se prolongará en caso de respuesta clínica lenta, que haya un inadecuado control de la fuente de infección o inmunosupresión incluida neutropenia (recomendación 1D). En caso de evidenciar una causa no infecciosa se suspenderá de inmediato el antibiótico con el fin de disminuir el riesgo de una infección posterior por un germen resistente al manejo iniciado. ${ }^{4}$ Los regímenes recomendados por IDSA y SIS se ven en la Tabla 4.
En nuestra experiencia el uso de antibióticos de amplio espectro para el cubrimiento de Gram positivos y negativos tipo carbapenémicos o piperacilina tazobactam son la preferencia. El seguimiento de la terapia mediante reportes del cultivo de líquido peritoneal de los lavados de la cavidad a repetición puede ser un arma de doble filo, pues la presencia de gérmenes no siempre significa falla terapéutica. Además, la penetrancia antibiótica y las propiedades farmacodinámicas son erráticas en estos casos y las concentraciones terapéuticas bactericidas pueden variar con mucha frecuencia..$^{38-40}$

\section{Manejo quirúrgico: principios}

La cirugía es el pilar fundamental del manejo en la sepsis de origen abdominal, ya que si falla todo el soporte no quirúrgico será insuficiente y el paciente fallece. ${ }^{1}$ El manejo quirúrgico de la peritonitis sigue cuatro principios:

1. Control de la fuente: ocluir la fuga del material infeccioso.

2. Drenaje: evacuar bacterias, pus y detritos.

3. Descompresión: manejo del síndrome de hipertensión abdominal.

4. Control de calidad: control del reparo y del drenaje.

El esquema estándar o inicial comprende el primero y segundo principios. El tercero y cuarto son posibles solo utilizando técnicas quirúrgicas específicas como la laparotomía STAR (staged abdominal repair), la laparotomía a demanda ola planeada. Conocer y entender en qué se basan y de qué se compone cada uno de los principios, brinda las herramientas para lograr un mejor desenlace clínico.

\section{Principio 1: Control de la fuente, ocluir la fuente de infección}

Cualquier peritonitis supurativa resulta de un foco en el cual hay fuga de material infeccioso hacia la cavidad abdominal que después se extenderá a otros órganos intraabdominales. Por lo regular el foco está en una víscera hueca que contiene bacterias como parte de su flora normal y se procederá a suturar, resecar o 


\section{Tabla 4. Regímenes antimicrobianos recomendados por IDSA y SIS}

\section{IDSA (Infectious Diseases Society of America)}

Infección leve a moderada

\section{SIS (Surgical Infection Society)}

\section{Paciente de bajo riesgo}

\section{Régimen de un solo agente:}

- Ampicilina-sulbactam

- Ticarcilina-clavulanato

- Ertapenem

- Cefotetan
- Cefoxitina
- Ertapenem
- Meropenem
- Impicilina-sulbactam
- Piperacilina-tazobactam
- Ticarcilina-clavulanato

\section{Régimen combinado:}

- Cefazolina o cefuroxima + metronidazol

- Fluoroquinolona + metronidazol
- Ciprofloxacina + metronidazol

- Aminoglucósidos + antianaerobio

- Aztreonam + clindamicina

- Cefuroxima + metronidazol

- Cefalosporina de $3^{\mathrm{a}}$ y $4^{\mathrm{a}}$ generación + antianaerobio

Infecciones muy severas Pacientes de alto riesgo

Régimen de un solo agente:

- Meropenem

- Imipenem-cilastatina

- Piperacilina-tazobactam

- Meropenem

- Imipenem-cilastatina

- Piperacilina-tazobactam

\section{Régimen combinado:}

- Ciprofloxacina +metronidazol

- Aztreonam + metronidazol

- Cefotaxime, ceftriaxona, ceftazidime, cefepime + metronidazol

- Ciprofloxacina +metronidazol

- Aminoglucósidos + antianaerobio

- Aztreonam + clindamicina

- Cefalosporina de $3^{\mathrm{a}}$ y $4^{\mathrm{a}}$ generación + antianaerobio.

Adaptado de:Solomkin JS, et al. Intra-abdominal Sepsis: Newer Interventional and AntimicrobialTherapies Clin Infect Dis. 2003;37:9971005; Mazuski JE, et al. for the Therapeutic Agents Committee of the Surgical Infections Society. The Surgical Infection Society guidelines on antimicrobial therapy for intra-abdominal infections: an executive summary Surg Infect (Larchmt). 2002;3:I6I-I73.

exteriorizar. La forma de reparar la lesión varía con la enfermedad, la localización anatómica (por ejemplo, las duodenales no pueden ser exteriorizadas), el grado de inflamación local y la respuesta generalizada, así como el estado previo del paciente, sus comorbilidades y reservas funcionales. A pesar de la tendencia actual de la anastomosis primaria en la víscera hueca, por la gran morbilidad de los abocamientos en los casos más complejos donde el compromiso inflamatorio amenaza una inapropiada cicatrización o un pobre desarrollo de la fuerza tensil, se recomienda exteriorizar, pues el drenaje así sea escaso hacia el interior de la cavidad, será fuente de perpetuación de la enfermedad. Es menos común la acumulación patológica de bacterias en órganos o espacios que son estériles (abscesos o empiemas) que pueden perforarse y causar peritonitis, como por ejemplo el tracto biliar o urinario. Todo absceso o empiema debe drenarse o removerse mediante cirugía, pues la terapia antibiótica sola no es igual de efectiva.

\section{Principio 2: Drenaje, evacuar el pus y sustancias adyuvantes de la infección}

El fluido infeccioso peritoneal (pus, fibrina, tejido necrótico, bacterias, factores de crecimiento) debe evacuarse de la cavidad abdominal y puede ser seroso, fibrinoso, bilioso, purulento o fecaloide. Aunque es un procedimiento muy conocido y utilizado, es importante recordar que el desbridamiento no debe ser tan radical, 
ya que esto lleva a altas tasas de complicaciones como son la formación de fístulas y sangrado de difícil control. ${ }^{2}$ Se harán irrigaciones con técnicas adecuadas y drenaje total de la cavidad, secando con cuidado para no alterar la defensa local del huésped. ${ }^{1.3}$ No se recomienda el uso de antisépticos locales por la toxicidad para el peritoneo. Los antibióticos locales son inoficiosos porque la concentración efectiva bactericida no se sostiene el tiempo suficiente después del lavado abdominal para lograr el control de la infección y por lo tanto son generadores de resistencia bacteriana. ${ }^{1,4}$

\section{Principio 3: Descomprimir, manejo del sindrome de hipertensión intraabdominal}

El peritoneo y su tejido conectivo pueden llegar a absorber hasta más de diez litros de líquido inflamatorio que se produzca en la cavidad abdominal. Recordemos que el aumento de producción de óxido nítrico genera un íleo que contribuye al incremento de la presión intraabdominal, la cual compromete la función cardiovascular, pulmonar, renal y hepática. Con frecuencia hay alteración del retorno venoso e hipoperfusión en el flujo esplácnico y la pared abdominal que compromete la oxigenación de los tejidos locales. Es por esta razón que en la cirugía inicial no se debe tratar de cerrar la aponeurosis a tensión pues complica el problema. Este síndrome compartimental abdominal debe manejarse con técnicas en que se deje el abdomen abierto o mediante laparotomía STAR. ${ }^{41-43}$

\section{Principio 4: Control de calidad, control del reparo y del drenaje}

La cirugía inicial a veces no es suficiente para verificar la integridad de las anastomosis realizadas, la viabilidad de un segmento intestinal o persistencia de la peritonitis. Estas complicaciones deben ser seguidas y manejadas en algunas ocasiones por largos períodos. Es mejor hacer una reexploración planeada cuando los problemas o complicaciones posoperatorios son esperados. Esto significa que la cavidad abdominal necesita ser reintervenida a las 24 horas, pues el crecimiento bacteriano después del lavado abdominal es rápido y se sabe que el número de bacterias será el mismo que 24 horas antes del lavado quirúrgico inicial. ${ }^{7}$

\section{Definiciones}

A continuación se aclararán algunas definiciones importantes de los procedimientos más utilizados en el manejo quirúrgico de la peritonitis secundaria con el fin de unificar conceptos y establecer un algoritmo de manejo.

\section{Cirugía inicial o índice}

El manejo quirúrgico de la infección intraabdominal debe basarse en los principios establecidos durante las dos primeras décadas del siglo XX. El doctor Kirschener y colaboradores en 1926 definieron la estrategia terapéutica de manejo de infecciones abdominales con tres postulados: control de la fuente de referencia en la cirugía inicial, manejo antibiótico temprano y soporte médico de la disfunción orgánica secundaria. ${ }^{6}$ Esto constituyó el estandar quirúrgico para el manejo inicial de los pacientes con infecciones intraabdominales durante las primeras décadas del siglo XX y redujo en forma significativa la mortalidad en másdel $90 \%$, comparado con el ratamiento no quirúrgico en cerca de un $40 \%$ de los casos. ${ }^{1,4}$

\section{Laparotomía a demanda}

Comprende el manejo tradicional de múltiples exploraciones de la cavidad abdominal luego del procedimiento quirúrgico, en el cual el cirujano decide cuándo reintervenir el abdomen basado en hallazgos clínicos y paraclínicos del estado de la infección.

\section{Relaparotomía planeada}

Consiste en decidiren la cirugía inicial cuando reexplorar al paciente de acuerdo con los hallazgos encontrados; en general se contempla dentro de los tres primeros días, independiente de la evolución del paciente en su posoperatorio inmediato. La decisión de reintervenir al paciente forma parte del plan inicial de manejo. Desde el punto de vista histórico, fue la isquemia mesentérica la primera patología quirúrgica en que se planteó lo que entonces se llamó una "segunda mirada" de manera planeada. Penninckx y colaboradores fueron los primeros en reportar la relaparotomía planeada en el contexto de infecciones intraabdominales. ${ }^{9}$ La razón para realizarla 
es lograr un control definitivo de la fuente cuando no se logró en la cirugía inicial y así realizar un nuevo lavado peritoneal con el fin de disminuir la respuesta inflamatoria sistémica y la falla multiorgánica que ocasionan los restos infecciosos dentro de la cavidad.

\section{Indicaciones de una relaparotomía planeada}

Las indicaciones para asumir la conducta de una relaparotomía planeada están mal definidas y en su mayoría basadas en experiencia clínica no sistemática. Estos son algunos de los argumentos más utilizados en la literatura:

- Falla en obtener un adecuado control de la fuente durante la cirugíaíndice. Esta es la más aceptada de las indicaciones. Ejemplos claros son la necrosis pancreática infectada o una fístula intestinal que no fue reparada en forma apropiada o exteriorizada.

- Necesidad de desbridar o drenar procesos infectados recurrentes mal localizados y de difícil acceso, como son en el retroperitoneo, la fascitis o una perforación de colon o duodeno.

- La peritonitis fecal es una indicación relativa, ${ }^{10,11}$ bajo la presunción de que la contaminación fecal masiva requerirá más de un procedimiento para lograr una adecuada limpieza de la cavidad.

- Inestabilidad del paciente durante la cirugía inicial que en ocasiones obliga a hacer un procedimiento abreviado tipo control de daños, con una subsecuente relaparotomía programada para finalizar el control de la fuente de infección y la limpieza de la cavidad. En caso que se haya dejado "empaquetado" al paciente, retirar las compresas implica un nuevo procedimiento. ${ }^{12}$

- El doctor Wittman y colaboradores ${ }^{13}$ proponen una política aún más agresiva combinando la relaparotomía planeada con una nueva técnica llamada laparotomía STAR (staged abdominal repair) a la cual nos referiremos más adelante, como una mejor opción para vigilar una anastomosis en el caso de que hubiese estadoindicada una colostomía. Esta última es la más discutida. ${ }^{4}$

\section{Laparostomía o abdomen abierto}

El término laparostomía, que implica dejar el abdomen abierto, fue acuñado por el doctor Fagniez de Paris. ${ }^{20} \mathrm{El}$ doctor Pujol fue el primero en sugerir que el abdomen con infección severa debía ser tratado abierto como si fuera un gran absceso dentro la cavidad. ${ }^{21}$ El concepto de que la peritonitis y su manejo quirúrgico inicial resultaban en un incremento de la presión intraabdominal fue siendo aceptado en forma paulatina y aplicado a lo largo del siglo XX; sin embargo, los beneficios de la prevención y manejo de la hipertensión abdominal con el uso de la laparostomía fueron establecidos y aceptados hace poco. ${ }^{20} \mathrm{El}$ uso de la laparostomía no elimina la necesidad de reexplorar el abdomen, pero la facilita. $\mathrm{Si}$ el abdomen va a ser revaluado en 48 horas ¿para qué cerrarlo todo?

\section{Indicaciones de laparostomía}

En la actualidad, está indicada cuando el abdomen no puede cerrarse o existe alto riesgo de desarrollo de hipertensión abdominal.

¿Cuándo el abdomen no puede ser cerrado?

- Luego de pérdidas tisulares mayores de la pared abdominal, secundarias a trauma o desbridamientos extensos en fascitis necrotizante (pérdida del domicilio).

- Gran edema de asas o del espacio retroperitoneal después de un trauma mayor, por resucitación con fluidos o cirugía mayor (ruptura de aneurisma de aorta abdominal).

- Cuando existe aponeurosis de mala calidad debido a múltiples laparotomías previas.

¿Cuándo el abdomen no debe ser cerrado?

- Cuando se toma la decisión en la cirugía inicial de reexplorar en las siguientes 72 horas. 
- Cuando el cierre de la pared abdominal es solo posible en condiciones de extrema tensión, comprometiendo la aponeurosis y desencadenando hipertensión abdominal o síndrome compartimental abdominal. ${ }^{4446}$

\section{Laparotomía STAR (staged abdominal repair)}

Se define como una serie de laparotomías realizadas cada 24 a 48 horas hasta lograr la limpieza de la cavidad peritoneal. ${ }^{47}$ haciéndolo con sumo cuidado pues las asas intestinales y la superficie peritoneal están edematosas, friables, y con tendencia a sangrar. Lo ideal es que el cirujano realice la laparotomía inicial sea el mismo que reintervenga o al menos que esté presente.

\section{Lavado peritoneal. ¿Cómo debe hacerse una relaparotomía o un lavado peritoneal?}

El objetivo de la relaparotomía programada o a demanda, es decir los lavados peritoneales que se realicen posteriores a la cirugía inicial, es el de drenar todas las colecciones infectadas y controlar las fuentes persistentes de contaminación dentro de la cavidad abdominal. Estos lavados quirúrgicos y su manera de hacerlos son muy individualizados y la decisión sobre la extensión de la exploración es crucial porque cuanto más amplia es, representa mayor peligro para las estructuras adyacentes. Los estudios no muestran resultados estadísticos significativos en cuanto a mortalidad con una u otra técnica, pero sí hay una gran diferencia en los costos a favor de la laparotomía a demanda que se hace de acuerdo con la evaluación y análisis clínico de estos pacientes. Esta es la técnica que más usamos en nuestro grupo y la que recomendamos. ${ }^{32,44}$

\section{Tiempo de la intervención}

Existe consenso universal y gran evidencia empírica de la importancia del tiempo en el manejo de la sepsis. Se sabe también que la demora en remover fuentes de contaminación aumenta la carga bacteriana, el índice de complicaciones y la mortalidad. ${ }^{6,48}$ Sin embargo, la velocidad de crecimiento, el inóculo bacteriano y su tasa de crecimiento o virulencia son factores que hasta ahora no se han podido predecir con claridad. Con base en esto se utilizan entonces desenlaces intermedios clínicos claros como son la severidad de los síntomas, la velocidad del deterioro clínico, el grado de disfunción orgánica y la severidad de la respuesta inflamatoria como marcadores para la toma de decisiones. Sin embargo, en el afán del control de la infección surgen otras complicaciones como son el trauma adicional por la exploración a ciegas no planeada. Este balance no permite hacer recomendaciones específicas en cuanto al tiempo entre intervenciones y hay que tomar decisiones en forma individual. $^{22}$

\section{Discusión}

La peritonitis secundaria es relevante por su alta mortalidad (20-60\%), estancia hospitalaria prologada y frecuente morbilidad, debido al desarrollo de sepsis y falla orgánica múltiple. ${ }^{23}$ La peritonitis secundaria corresponde a cerca de 9.3/1.000 de las admisiones a urgencias en los Estados Unidos. ${ }^{48}$ Además, un número importante de pacientes (12-16\%) que son llevados a cirugía electiva desarrollan peritonitis en el posoperatorio. ${ }^{19,20} \mathrm{La}$ utilización de los recursos de atención en salud en el manejo de los pacientes con peritonitis secundaria es extensa, requiriendo cirugía para eliminar el foco de la infección y atención multidisciplinaria en laUCI. Después de la laparotomía inicial, puede ser necesaria una reintervención para eliminar la peritonitis persistente o el foco. Existen dos estrategias con respecto a las relaparotomías: cuando la condición del paciente lo exige ("a demanda")y la reintervención programada. El objetivo de la primera es llevar a cabo la reoperación solo en quienes es probable que se beneficien de esta cirugía, como aquellos con deterioro clínico o que persisten sin mejoría a pesar del tratamiento y el manejo adecuados. En la estrategia programada se realiza una relaparotomía cada 36 a 48 horas para la revisión, drenaje y lavado peritoneal de la cavidad abdominal hasta que los resultados son negativos para la peritonitis en curso. En ocasiones facilita la detección temprana de peritonitis persistente 0 descubre nuevos focos infecciosos, pero alberga el riesgo potencial de reexploraciones innecesarias en pacientes críticos, mientras que la relaparotomía a demanda puede llevar a un retraso en potencia perjudicial para la detección de infecciones en curso. ${ }^{49}$ 
Los puntos cardinales del tratamiento quirúrgico de las infecciones intraabdominales son:

1. Eliminar la fuente de contaminación.

2. Reducir el inóculo bacteriano.

3. Prevenir el desarrollo de sepsis persistente.

No es claro, a luz de la presente revisión, cuál es la técnica ideal para manejar estos enfermos. Lo que sí parece fundamental es mantener los principios de manejo integral descritos desde comienzos del siglo pasado. Nunca será suficiente enfatizar sobre la reanimación temprana, el control adecuado y oportuno de la fuente y el uso racional de antibióticos como las estrategias fundamentales para el manejo de estos enfermos. ${ }^{13,46,50}$

\section{Conclusiones}

El principal punto de controversia en el manejo actual de los pacientes críticos con sepsis abdominal se encuentra en el uso de dos aproximaciones de relaparotomía: a demanda y programada. La evidencia acumulada hasta ahora es la siguiente:

1. No existen ensayos clínicos aleatorios de comparación de las dos aproximaciones.

2. Se conocen dos metanálisis de estudios observacionales analíicos de baja calidad, muchos rerospectivos en su recolección con gran heterogeneidad entre ellos. Estos son los hallazgos:

- Los estudios iniciales reportaron mejores resultados con el uso de laparotomías con pobre calidad de ejecución y poca precisión en los resultados.

- Estudios más recientes y en general con calidad en su ejecución y recolección, muestran mejores resultados con el uso de la técnica a demanda. Ante estos hallazgos concluimos que no existe evidencia suficiente para hacer una recomendación definitiva. La evidencia apunta más a hacer intervenciones a demanda.

\section{Referencias}

1. Solomkin JS, Mazuski JE. Intra-abdominal sepsis: newer interventional and antimicrobial therapies. Infect Dis Clin N Am. 2009; 23 (3): 593--608.

2. Ordoñez C, Puyana J. Management of peritonitis in the critically ill patient. Surg Clin N Am. 2006 Dec; 86 (6): 1323-49.
3. Schein M, Marshall J. Source control for surgical infections. World J. Surg 2004: $28,638-45$.

4. Mazuski JE, Sawyer RG, Nathens AB, DiPiro JT, Schein M, Kudsk KA, Yowler C. The Surgical Infection Society guidelines on antimicrobial therapy for in a-abdominal infections: an executive summary. Surg Infect (Larchmt). 2002; 3 (3): 161-73.

5. Penkoske P, Buchman TG. The relationship between the surgeon and the intensivist in the surgical intensive care unit. Surg Clin N Am. 2006 Dec; 86 (6): 1351-57.

6. Schecter WP, Ivatury RR, Rotondo MF, Hirshberg A. Open abdomen after trauma and abdominal sepsis: a strategy for management. J Am Coll Surg. 2006 Sep;203(3):390-6

7. Calandra T, Cohen J. The intemational sepsis forum consensus conference on definitions of infection in the intensive care unit. Crit Care Med. 2005 Jul; 33(7):1538-48.

8. Tellado JM, Christou NV, editors. Intra-abdominal infections. Madrid, Spain: Harcourt; 2000.

9. Dellinger RP, Levy MM, Carlet JM, Bion J, Parker MM, Jaeschke R, et al Surviving sepsis campaign: intemational guidelines for management of severe sepsis and septic shock: 2008. Crit Care Med. 2008 Jan; 36(1):296-327.

10. Solomkin JS, Mazuski JE, Baron EJ, et al. Guidelines for the selection of anti-infective agents for complicated intra-abdominal infections. Clin Infect Dis. $2003 ;$ 37(8): 997-1005.

11. Malangoni MA. Current concepts in peritonitis. Curr Gastroenterol Rep. 2003 Aug; 5(4):295-301.

12. Chromik AM, Meiser A, Hölling J, Sülberg D, Daigeler A, Meurer K, et al Identification of patients at risk for development of tertiary peritonitis on a surgical intensive care unit. J Gastrointest Surg. 2009 Jul;13(7):1358-67.

13. Wittmann DH, Schein M, CondonRE. Management of secondary peritonitis. Ann Surg. 1996 Jul; 224(1): 10-18.

14. Deitch EA. Role of the gut lymphatic system in multiple organ failure. Curr Opin Crit Care. 2001 Apr;7(2):92-8.

15. Pinsky MR, Matuschak GM. Multiple system organ failure host defense homeostasis. Crit Care Clin. 1989 Apr; 5(2):199-220

16. Lamme B, Mahler CW, van Ruler O, Gouma DJ, Reitsma JB, Boermeester MA. Clinical predictors of ongoing infection in secondary peritonitis: systematic review. World J Surg. 2006 Dec;30(12):2170-81 .

17. Gotzinger P, Wamser P, Barlan M, Sautner T, Jakesz R, Fugger R. The surgical concepts of planned and on-demand reoperation in the treatment of diffuse intra-abdominal infection. Acta Chir Austriaca. 2000; 32 (4):167-70.

18. Wittmann DH. Intra-abdominal infections. Introduction. World J Surg. 1990 Mar-Apr; 14(2):145-7.

19. Schein M. Planned reoperations and open management in critical intraabdominal infections: prospective experience in 52 cases. World $\mathbf{J}$ Surg. 1991 Jul-Aug; 15(4): 537-45.

20. Wittmann DH. Staged abdominal repair: development and current practice of an advanced operative technique for diffuse suppurative peritonitis. Acta Chir Austriaca. 2000; 32(4):171-78.

21. Wittmann DH. The colon. In: Scheim M, Marshall JC, editors. Source control, a guide to the management surgical infections. New York: Springer - Verlag;2003.p. 103-108. 
22. Gómez M. Manejo del trauma abdominal en tópicos en cuidado intensivo. Trabajo presentado en el primer Consenso Nacional en Cuidado Intensivo; 1999 mayo 16; Cartagena de Indias, Colombia. p 136.

23. Gómez M, Cruz L, Villabón M. Hipertensión intraabdominal y síndrome compartimental. En: González MA. Fundamentos de medicina : paciente en estado crítico. 3a ed. Medellín: Corporación para Investigaciones Biológicas; 2003. p. 180 .

24. van Ruler O, Mahler CW, Boer KR, Reuland EA, Gooszen HG, Opmeer $\mathrm{BC}$, et al. Comparison of on-demand vs planned relaparotomy strategy in patients with severe peritonitis: a randomized trial. JAMA. 2007 Aug 22; 298(8):865-72.

25. Padilla T, Fustinoni O. Sindromes clínicos en esquemas. $17^{\mathrm{a}}$ ed. Buenos Aires : El Ateneo; 2000. p. 33.

26. Schein M, Wittmann DH, Aprahamian CC, Condon RE. The abdominal compartment syndrome: the physiological and clinical consequences of elevated intra-abdominal pressure. J Am Coll Surg. 1995 Jun; 180(6):745-53.

27. Pujol JP. Moderate hypertension defined as sustained elevation of 21-35 mmHg. Paris: U.E.R.X Bichat; 1975.

28. Gómez M, Posada C, Cruz LE. Soporte nutricional en cuidado intensivo experiencia de 4 años. Acta Colomb Cuid Int. 2001; 4(2): 109.

29. Fry D. Antibiotic kinetics in febril multiple system trauma patients. In: Cunha BA, editor. Infectius diseases in critical care medicine. London: Informa Healthcare; 2010. p 521-35.

30. Gomez E. Trastornos en la hoemostasis de los liquidos. En: Ordoñez CA, Ferrara R, Buitrago R. Cuidado Intensivo y trauma. Bogotá: Distribuna; 2009. p 201

31. Hutchins RR, Gunning MP, Lucas DN, Allen-Mersh TG, Soni NC. Relaparotomy for suspected intraperitoneal sepsis after abdominal surgery. World J Surg. 2004 Feb;28(2):137-41.

32. Koperna T, Schulz F. Relaparotomy in peritonitis: prognosis and treatment of patients with persisting intraabdominal infection. World J Surg. 2000 Jan;24(1):32-7.

33. Aydin C. Aytekin FO, Yenisey C, Kabay B, Erdem E, Kocbil G. The effect of different temporary abdominal closure techniques on fascial wound healing and postoperative adhesions in experimental secondary peritonitis. Langenbecks Arch Surg. 2008 Jan; 393(1):67-73.

34. Kirshtein B, Roy-Shapira A, Lantsberg L, Mizrahi S. Use of the "Bogota bag" for temporary abdominal closure in patients with secondary peritonitis.. Am Surg. 2007 Mar;73(3):249-52.

35. Schecter WP, Ivatury RR, Rotondo MF, Hirshberg A. Open abdomen after trauma and abdominal sepsis: a strategy for management. J Am Coll Surg. 2006 Sep;203(3):390-6.
36. Enciso Olivera CO, Guerra Urrego KA, Gómez Duque M, Meneses Góngora E. Costos de atención en UCI de un hospital universitario de Bogotá D. C. Repert. Med. Cir. 2006; 15(3): 133-42.

37. Schöffel U. Timing of intervention. In: Schein M, Marshall JC. Source control: a guide to the management of surgical infections. New York: SpringerVerlag; 2002. p. 279-87.

38. Lamme B, Boermeester MA, Reitsma JB, Mahler CW, Obertop H, Gouma DJ. Meta-analysis of relaparotomy for secondary peritonitis. Br J Surg. 2002 Dec; 89(12):1516-24.

39. Agalar F, Eroglu E, Bulbul M, Agalar C, Tarhan OR, Sari M. Staged abdominal repair for treatment of moderate to severe secondary peritonitis. World J. Surg. 2005 Feb; 29(2): 240-44.

40. Anaya DA, Nathens AB. Risk factors for severe sepsis in secondary peritonitis. Surg Infect (Larchmt). 2003; 4(4):355-62.

41. Van Goor H. Interventional management of abdominal sepsis: when and how. Langenbecks Arch Surg. 2002;387(5-6): 191-200.

42. Adkins AL, Robbins J, Villalba M, Bendick P, Shanley CJ. Open abdomen management of intra-abdominal sepsis. Am Surg. 2004 Feb;70(2):137-40

43. Manejo de la peritonitis y la sepsis intraabdominal en cuidado intensivo. En: Ordoñez CA, Ferrada R, Buitrago R, editors. Cuidado intensivo y trauma. Bogotá: Distribuna; 2003. p. 667-84.

44. Rakic M, Popovic D, Rakic M, et al. Comparison of on-demand vs planned relaparotomy for treatment of severe intra-abdominal infections. Croat Med J 2005:46(6):956-63.

45. Sihler $\mathrm{KC}$, Nathens $\mathrm{AB}$. Management of severe sepsis in the surgical patient. Surg Clin NorthAm. 2006 Dec;86(6): 1457-81.

46. Christou NV, Barie PS, Dellinger EP, et al. Surgical Infection Society Intra Abdominal Infection Study:prospective evaluation of management techniques and outcome. Arch Surg 1993;128(2): 193-8.

47. Marshall JC, Christou NV, Meakins JL. The gastro intestinal tract: the "undrained abscess" of multiple organ failure. Ann Surg.1993; 218(2):111-9.

48. Borraez OA. Abdomen abierto. En: Quintero Hernández GA, Nieto JA, Lerma $\mathrm{CH}$, editores. Infección en cirugía. Bogotá: Médica Panamericana; 2001. p. 230-37.

49. Ozgüç H, Yilmazlar T, Gürlüler E, Ozen Y, Korun N, Zorluoðlu A. Staged abdominal repair in the treatment of intraabdominal infection: analysis of 102 patients. J Gastrointest Surg. 2003; 7(5):646-51.

50. Biswajit M. Staged abdominal repair (STAR) operation: how I did it. Indian J Surg 2004; 66(3): 182-4. 